\title{
Public policies for wellbeing with justice: A theoretical discussion based on capabilities and opportunities
}

\author{
Jaya Krishnakumar · Ricardo Nogales
}

\begin{abstract}
This article presents a theoretical framework that combines virtues and strengths of the Capability Approach (CA) and Equality of Opportunity (EOp) approach for analyzing public policies that aim to improve individual wellbeing and social justice. We show that neither approach is sufficient on its own for this goal. It is particularly useful to combine the two approaches because the CA offers a positive way of thinking about what wellbeing is, while the EOp Approach provides more formal insights on how to configure public policies to achieve social justice and increase individual wellbeing from a normative perspective. We make the case that EOp in its original (ex-post) conception is too heavily centered on lifestyle outcomes and oblivious to individual heterogeneity. However, we argue that it contains elements that are compatible with the CA rationale from an ex-ante point of view. Individual efforts play a crucial conceptual role in our proposed combination because they influence and are influenced by individual capabilities. Our optimal policy for improving wellbeing with EOp is one that aims to equalize expected capabilities across different groups, characterized by circumstances, through a maximin algorithm. We provide a technical analysis of our optimal policy taking into account the influence of circumstances and policies on efforts and capabilities.
\end{abstract}

Keywords: Capability Approach, equality of opportunity, individual wellbeing, public policies.

\section{Introduction}

"If one is to intervene, then the significant life chances that people have constitute a key variable on which the State should focus" (Anand et al., 2005; p4 including emphasis).

Today, the increase of collective wellbeing combined with justice is perceived as an essential ingredient of any development paradigm and it is not an exaggeration to say that its importance is steadily increasing. Perhaps an interesting reflection of the consensus that this paradigm enjoys in the international arena is the UN's set of Millennium Development Goals (MDG; Kabeer, 2010), which not only advanced the idea that wellbeing is multidimensional in nature but also included some aspects of equality and fairness among its goals. All current dialogues on the post2015 agenda, such as the 2013 Rio+20 summit, the 2014 Beyond 2015 meeting of CSO's in South Africa and the UN's High Level Panel on post-2015 Development Agenda, are converging towards the need for emphasizing even further in future agendas the sustainability and social justice dimensions of development. Even in countries that currently articulate public policies around some seemingly alternative conceptions of development, such as Bolivia with its Vivir Bien (Ministerio de Planificación del Desarrollo, 2006), Ecuador with its Buen Vivir (SENPLADES, 
2009) or Bhutan with its Gross National Happiness (Ura, Alkire, Zangmo \& Wangdi, 2012), the improvement of collective wellbeing and its just distribution remains the common goal.

The goal may be a common one, but of course the means prescribed by governments and international organizations are different because needs, priorities, contexts and concerns are also different. Tax regime changes, conditional transfers, offers of public health services and goods, better nutrition, improvements in education and social security regulations are among the various recipes utilized to allow people to achieve better states of wellbeing and social justice. The natural heterogeneity of societies has always forced discussions around appropriate ways to foster wellbeing through public policies to be quite controversial (see for example Ravallion, 2010, 2012).

There are, however, some international theoretical agreements upon such issues of a normative nature. Many modern internationally accepted frameworks for the assessment of wellbeing are grounded on the Capability Approach (Sen, 1985, 1999, abbreviated CA), which is deeply influential amongst development scholars and in political spheres. Well-known initiatives such as UNDP's Human Development Index (Anand \& Sen, 1993; Haq, 1999; Sen, 1999), OPHI's Multidimensional Poverty Index (Alkire \& Foster, 2011) and the MDGs have been theoretically linked to the CA. The CA has also been the theoretical stepping-stone for research on novel methodologies for its operationalisation in recent years (see e.g. Anand, Krishnakumar \& Tran, 2010; Anand, Santos \& Smith, 2007; Anand et al., 2009 ; Kuklys, 2005; Krishnakumar, 2007; Krishnakumar \& Ballón, 2008; Simon et al., 2013). This approach is rightfully praised for its positive way of thinking about what individual wellbeing is (Sen 1985, 1999, 2009); its contribution to policies for improving wellbeing is much more humble. On this matter, Robeyns (2003) states,

"... the CA is an approach to interpersonal comparisons which argues for functionings and capabilities as the relevant evaluative space, where each application (be it theoretical or empirical) can, and probably has to, be supplemented with other theories. These other theories are normative theories (for example a normative theory of choice or a theory on the normative relevance of class, gender or race), which are in turn based on positive theories of human behavior and agency and societal process." (pp. 45-46, emphasis own).

Although powerful, the theoretical approach that is the core foundation of modern wellbeing analyses was not conceived to give insights about ways to improve wellbeing with justice through policymaking. The CA does not explicitly advocate for any specific public intervention or algorithm in the quest for increased social justice and hence it is not a normative theory in that sense. In fact it strongly recommends a context and time-dependent assessment of the relevant wellbeing dimensions and their relative importance (Anand, Hunter \& Smith, 2005; Arneson, 1989; Robeyns, 2006). However, when thinking of public policies, supplementing the CA is an issue that should not be left lingering. Inspired by Fleurbaey (2007), we make the case that when aiming at fostering wellbeing with justice, policymakers inevitably face two fundamental questions:

1) While pursuing increases in collective wellbeing with justice, what is it that needs to be less unequal?

2) Amongst a very broad set of feasible situations, which social states are better/more just and should be promoted by public action?

Answers to both questions are needed for effective policymaking. However, these answers are never straightforward, because the very concepts of wellbeing and social justice are intrinsically normative and tightly linked to ethical reasoning. It is such a complex matter that, as we have established, one single theory, even as sound as the CA, has proven incapable of giving precise 
answers to both questions. Following the above quotation from Robeyns, the CA goes as far as bringing about an answer to the first question that we have raised, in the way of clearly stating that reduction (even elimination) of capability deprivation is required for a less unequal state of affairs.

There is, however, another influential theoretical framework for policymaking, namely the Equality of Opportunity Approach (Roemer 1998, abbreviated EOp), that has rapidly gained recognition over recent years and that has the potential of bringing a sound answer to the second question that we have raised. The EOp offers well established formal insights on what should be done in order to achieve social justice from a normative perspective, saying that one needs to eliminate the influence of circumstances beyond one's control in the process of achieving a desired outcome. This approach, however, has always been rather nuanced around what individual wellbeing is (Vellentyne, 2005), so it seems to have the potential of bringing about an answer to the second question only. For an operationalisation of EOp in the context of optimal taxation see Roemer et al. (2003); for evaluations of public policies for children's health see Jacquet and Van de Gaer (2011) and Van de Gaer, Vandenbossche \& Figueroa (2013); for an identification of fair and unfair income inequalities in Latin America see Bourguignon, Ferreira and Menéndez (2007) and Ferreira and Gignoux (2008).

Thus we see powerful complementarities between the two approaches, as one excels where the other one comes short. There are still few attempts to build a framework combining CA and EOp for analyzing policies for wellbeing at the empirical level: two recent attempts are Krishnakumar and Wendelspeiss (2011) and Wendelspeiss (2013). One has to acknowledge that there are notable similarities between the two approaches (Vallentyne, 2005; Igersheim, 2006), which have often led scholars to perceive that they provide similar elements of analysis regarding wellbeing and social justice and do not advance a theoretical combination. However, as stated by Chiappero Martinetti ${ }^{1}(2009)$, there are also substantial conceptual and practical differences between the two approaches, as the mechanisms that lead to personal lifestyle outcomes and wellbeing, and their possible links to public policies, are quite different from one theory to the other.

In this paper, we defend the idea that combining these theories sheds new and useful light to understand wellbeing and its relation to public policies because of the way they complement positive and normative reasoning, giving answers to both our questions. Throughout this document, we make the case that neither CA nor EOp, on its own, presents a combination of wellbeing and justice that can provide formal policy guidelines for improving wellbeing with justice through an appropriate optimality principle. We present arguments in search of clear answers to both our questions and show that an ordered integration of elements from both approaches leads to a plausible solution.

The paper is structured as follows: in section 2 we present some basic statements of Sen's and Roemer's theories, briefly recalling their essentials. In sections 3 and 4 we analyze plausible responses to the two questions raised in these introductory paragraphs, respectively, around the $\mathrm{CA}$ and the EOp approach. In section 5 we propose a theoretical combination as well as a technical derivation of our optimal policy and illustrate its usefulness. Section 6 concludes.

1 Chiappero Martinetti analyzes differences and similarities between the two theories but never attempts a combination. 


\section{Brief Review of Basic Statements of Sen and Roemer}

In modern development economics literature, Sen's and Roemer's theories are often categorized within the same framework of wellbeing and social justice contributions (Vallentyne, 2005). Indeed, there are clear similarities between them. They both have origins in criticism of utilitarianism and a monetary approach to wellbeing, i.e. only consideration of personal satisfaction or wealth as measures of personal wellbeing, and coincide in advocating that attention be paid to other aspects that make up an individual's life, such as the nature of her occupation, living conditions, health status, education, the social and institutional setup, etc. Both theories have philosophical origins in Rawls' principles for social justice (Rawls 1971; 1999): everyone is entitled to an adequate set of liberties, so long as they are compatible with the same liberties for others; and everyone is entitled to a fair equality of opportunities. They also coincide in acknowledging that one should go beyond outcome indicators and rather look at the underlying choice sets and circumstances.

However, the CA's strength relies in helping to understand better what individual wellbeing is, i.e. it is a positive theory of wellbeing, whereas the EOp contributes greatly to the ways for improving individual wellbeing with justice, i.e. it is a normative theory of policymaking. Let us briefly recall the fundamentals of each theory to support this idea and bring out the different elements of an answer to our guiding questions.

\subsection{Essentials of the Capability Approach}

Sen's CA has become a very important theory in modern economic studies, defining what we currently understand as human development (see for example, Anand et al., 2005). In this approach, human development and wellbeing are related to the sets of doings and beings from which a person has the ability to choose; the notions of liberty and freedom are of paramount importance within Sen's theory. In order to understand the richness and the broad way of thinking offered by the CA, it is key to briefly recall the distinctions among the different key concepts of Resources/Means, Functionings and Capabilities.

Functionings are life states and activities, objective and subjective (Vallentyne, 2005; Alkire, 2013), that are willingly and freely chosen, given a set of means and resources as well as social, political, family and cultural conditions. Capabilities are the set of all potential (feasible) functionings from which a person can freely chose. Thus, capabilities determine achieved functionings by a process of free choice by the individual in a particular setting. Capabilities are essentially unobservable, since they regroup possible non-materialized functionings with the effectively realized ones. Within this approach, an individual's wellbeing is increased when her capabilities are increased, as the latter have an intrinsic value in terms of the freedom to choose a valued life.

Capabilities are personal and individually formed by 'converting' the means and resources a person has, which include social and cultural characteristics, public/private endowments, commodities, services, norms, etc. (Comim, 2001). The conversion process for the internalization of these resources and means is personal and heterogeneous (Chiappero Martinetti \& Salardi, 2008).

Thus, the approach is essentially ethically individualistic (Robeyns, 2006) in the sense that it puts the human being's freedom to choose at the heart of any wellbeing assessment, over and above considerations at a group level or governmental actions.

Figure 1 below presents a diagram that summarizes the above statements regarding the process of construction of an individual's wellbeing within the CA. The capability set for a generic individual $i$ is formally expressed as follows (Sen, 1985): 


$$
Q_{i}=\left\{b_{i} \mid b_{i}=f_{i}\left(c_{i}\left(x_{i}, z_{i}\right)\right)\right\}
$$

with the notations being defined in the diagram below.

Figure 1: Capability Approach in a diagram

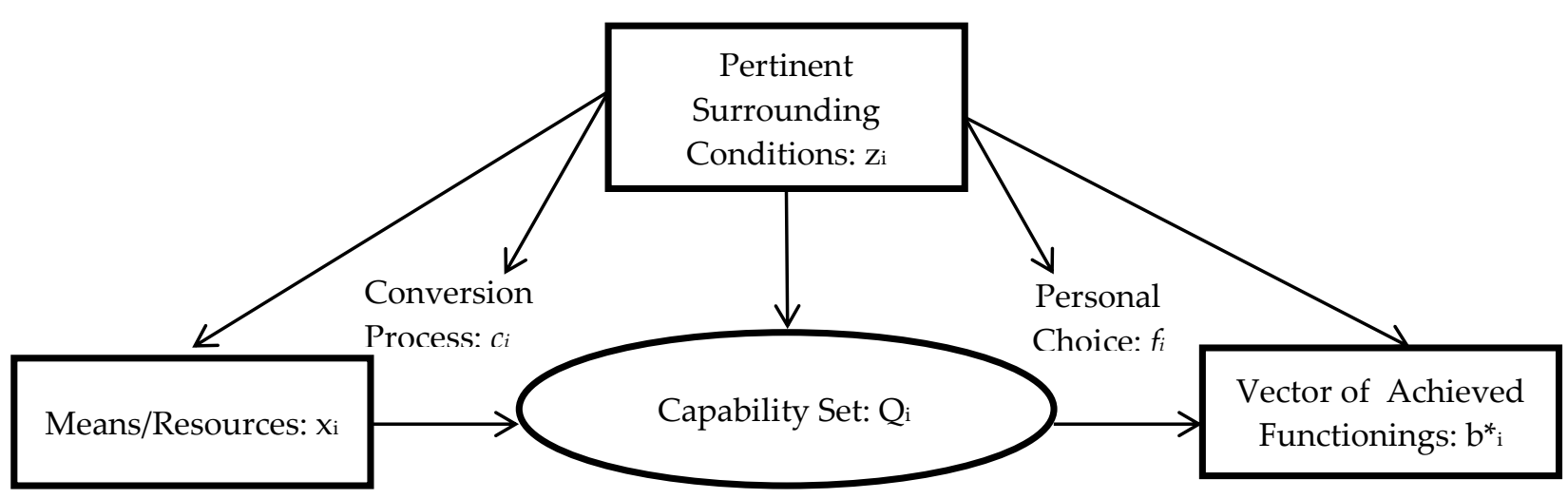

We denote as $\mathrm{b}^{*}$ the vector of achieved functionings, which is a particular vector-element of $Q_{i}$ chosen by the individual.

The approach's concentration on individual heterogeneity is evident and marks one of its most celebrated contributions to social justice literature. Nevertheless, the approach does not fail to recognize the importance of social constructions and relations for the whole process leading to the determination of these freedoms, such as legal rights, family history, culture and religion. Public policies are a part of these pertinent surrounding conditions that shape an individual's wellbeing, yet, as we have shown, the CA is almost silent concerning what kind of public actions are better for increasing individual wellbeing, while fostering social justice at the same time.

\subsection{Essentials of the Equality of Opportunity Approach}

Roemer's EOp approach has become a recognized pillar of normative public policymaking and assessment. It has introduced a way of thinking about public policy that is coherent with economic development 'with justice', promoting the equalization of life chances amongst individuals, arguing that an individual's lifestyle should be a function of her effort and choices, but not of characteristics she cannot or could not control.

Indeed, the EOp approach builds on the differentiation of aspects that are beyond an individual's control but influence her lifestyle outcomes or advantages, called circumstances, from aspects that have their origin in autonomous and willingly taken decisions that also exert such an influence, which Roemer proposes to call efforts. In the EOp approach, people sharing the same circumstances are grouped within a type. The lifestyle outcomes observed in a type constitute the achievable advantages for any member of the type and constitute their - common - Opportunities Set. Within a type, differences in outcomes can be attributed only to differences in efforts and constitute ethically acceptable differences between personal lifestyles.

Although not originally proposed by Roemer (1998), many groundbreaking empirical operationalizations of the EOp approach have successfully proven that one needs to take into account the fact that individual effort is significantly influenced by circumstances (Bourguignon et al., 2007; Ferreira \& Gignoux, 2008). Building on Bourguignon et al. (2007), we propose the schematic representation of the EOp approach given in Figure 2 below for a generic individual $i$. 
Figure 2: Equality of Opportunity Approach in a diagram

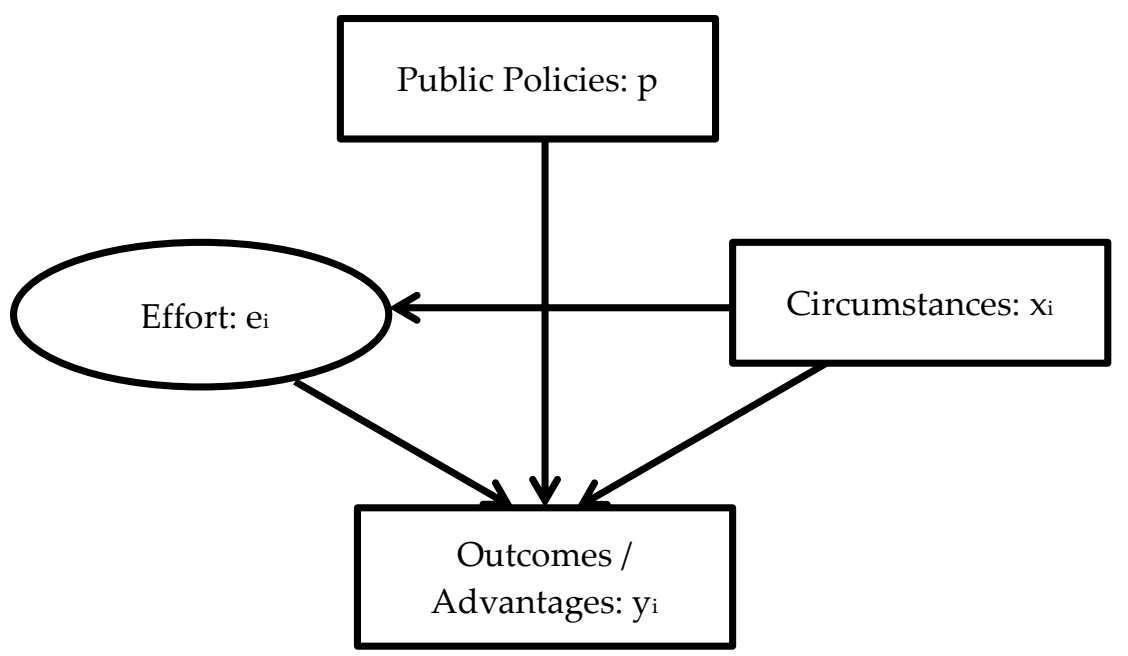

The diagram in Figure 2 can be translated into the following system of equations:

$$
\begin{gathered}
y_{i}=f\left(x_{i}, e_{i}, p\right) \\
e_{i}=g\left(x_{i}\right)
\end{gathered}
$$

Within a given type $t=1 \ldots T$, characterized by some particular circumstance variables say, gender or ethnicity, advantages $\mathrm{y}_{\mathrm{i}, \mathrm{t}}$ are a function of the degree or amount of effort exerted $\mathrm{e}_{\mathrm{i}, \mathrm{t}}$ and public policies $p_{\mathrm{t}}$, which could be thought to be type specific (Roemer, 1998) i.e. $y_{i, t}=y_{t}\left(e_{i, t}, p_{t}\right)$. As effort is hard to observe directly, one can consider a distribution of efforts within each type $t$ and divide it into centiles, identifying the level of effort, or relative effort, exerted by each individual in this type compared to all the other individuals within the same type. Since, within a type, differences in outcomes are attributable only to difference in degree of effort exerted, the effort distribution is conceptually merged to that of the outcomes of the type. Because of this perfect correspondence, it is possible to represent the outcomes of type $t$ as $y_{i, t}=y_{t}\left(\pi, p_{t}\right)$, where $\pi$ is a centile of the effort distribution.

The EOp advocates for the recognition of the ethical fact that an individual should not be held responsible for what is beyond her control and/or choice; thus individual responsibility and absence of impact of circumstances beyond control are put upfront in the assessment of a person's lifestyle (Igersheim, 2006). It is around this recommendation that the EOp approach has managed to develop a practical and logical formalization for policymaking yielding several empirical studies on optimal public interventions for social justice (Cogneau \& Gignoux, 2008; Jacquet \& Van de Gaer, 2011; Van de Gaer et al. 2013; Roemer et al., 2003). However, we have established that, while concentrating on normative aspects, this approach does not present an enriched positive concept of individual wellbeing.

\section{While pursuing increased collective wellbeing with justice, what is it that needs to be less unequal?}

Evidently, this question is far from being novel, but different answers are still in debate (cf. e.g. Ruggeri-Laderchi, Saith \& Stewart, 2003). Amongst others, in 1980, Sen had famously analyzed it in one of his most influential academic pieces, "Equality of What?" and within his remarkable series of academic contributions a path-setting answer was given: capabilities.

From the beginning, Sen's concern was linked to his disagreement with a somewhat tacit public consensus around the articulation of public policies seeking to equalize perceptions of 
individual satisfaction, as mandates utilitarianism and/or the equalization of basic goods (which include individual liberties and material resources), as suggested by Rawlsian prescription. According to Sen, the concept of equality of resources fails to take into account differences amongst individuals in terms of needs, desires and abilities, which, as we have stressed before, he considers to be fundamental for understanding individual wellbeing and, ultimately, social justice. Indeed, for Sen, the concept of individual wellbeing is far too complex and rich to be merged solely with the concepts of wealth or utility (satisfaction) and, above all, to be considered one-dimensional.

Among development scholars and policymakers, Sen's ideas have greatly overpowered other approaches to wellbeing, such as the monetary approach that sees consumption capacities as a synonym of wellbeing (Ruggeri-Laderchi et al., 2003) and the neoclassical welfarist point of view that focuses on the concept of utility, synonym of individual perception of satisfaction, thus as wellbeing (Blackorby \& Bossert, 2008; Schokkaert, 2007). Although consumption capacities are an important part of wellbeing and the concept of utility, being the basic pillar of neoclassical microeconomics, constitutes one of the most useful constructions in welfare economics, it is clear that they have important limitations for wellbeing and social justice analyses (Alkire \& Foster, 2011). Neither concept pays attention to the underlying factors from which those rational choices emerge because they are essentially one-dimensional (Robeyns, 2006), thus, offering a 'thin informational basis' (Anand et al., 2009) for wellbeing assessment. Sen has contributed greatly to today's consensus that wellbeing is essentially multidimensional (Alkire \& Foster, 2011; Ravallion, 2010, 2012; Ura et al., 2012).

By virtue of the $\mathrm{CA}$, it is now accepted that personal capability sets should be the measure of individual wellbeing; but the latter is difficult to directly observe given its counterfactual nature. In general, studies on the measurement of capabilities fall under two categories: a) studies that directly measure capability sets through questionnaires on people's choices and functionings (e.g. Anand et al., 2005, 2009; Anand \& van Hees, 2006), and b) studies that consider the observed functionings as partial manifestations of the capability set and resort to latent variable methodology for making inferences on capability sets using the observed indicators as well as other exogenous determinants of capabilities (e.g. Anand et al., 2010; Di Tommaso, 2007; Krishnakumar, 2007; Krishnakumar \& Ballón, 2008).

The same richness in perspective regarding individual wellbeing is not explicit in Roemer's work, nor is there an explicit recognition of lifestyle heterogeneity. Thus the search for equality of opportunity in the original sense of Roemer (1998) does not provide suitable elements for answering the question at hand in this section. Let us present the following arguments supporting this affirmation.

First, even if the EOp is as critical as the CA of utilitarianism as a theoretical basis for appraising social justice, it has mainly tended to consider one-dimensional outcomes such as education, health or wealth, except for a very few recent empirical operationalizations with multidimensional observable outcomes. Perhaps some of the most prominent amongst the latter are Yalonetzky's (2012) efforts to create a Dissimilarity Index for inequality assessment, Wendelspeiss' (2013) multidimensional latent variable approach and Brunori, Ferreira \& Peragine's (2013) international comparisons of inequality of opportunity. These exceptions aside, the vast majority of empirical applications of EOp follow the traditional Romerian flow by proposing one specific outcome for equalization in the quest for social justice and only one at a time (Igersheim, 2006).

Second, we perceive a clear distinction between the concepts of opportunity and capability. On the one hand, as suggested by the EOp, an opportunity is a situation that presents itself to an 
individual making use of which, and depending on her own efforts and skills, she will be able to achieve a certain outcome or lifestyle. Thus opportunities are not individual but common to a group of individuals that share the same circumstances (i.e. to each type in Roemer's terminology). On the other hand, the CA stresses that the concept of capability is unequivocally individual and personal. The concepts of opportunity and capability, therefore, operate at different levels of measurement and only the latter tend to take into account individual heterogeneity.

Third, according to the EOp, every member of a certain type can seize opportunities without distinction between each other. In effect, the only difference in terms of outcome between individuals of the same type is their effort and therefore, their own responsibility (Roemer, 1998). However, building on the CA, we stress that each individual always seizes opportunities differently from another, and that each individual personally (although, also influenced by factors other than personal) forms her own set of capabilities.

Let us close this section illustrating the superiority of the CA to answer our first question by virtue of its multidimensional and individually heterogeneous conception of wellbeing. In practice, it is usual to target a specific aspect of wellbeing when designing and implementing a public policy. However, spillovers around the target are undeniable and should never be left aside, for to do so would imply a drastic underestimation of the impact and blur possible ways of improving or correcting the policy. Take for instance a public policy aiming to improve nutrition of children aged $0-6$, such as a complementary nutrition program. The impacts of such a policy go far beyond the children's nutrition status as measured, for instance, by ratios combining variables such as height, weight and age; it enhances learning possibilities, recreational capacities and opportunities to develop talents in sports, music and so on, all of which are unobservable aspects of the children's wellbeing and all of which will always be imperfectly captured, even when resorting to multidimensional observable outcomes assessment. Furthermore, the children's wellbeing is undeniably influenced by the characteristics, values, culture and habits of their households and few would agree these important characteristics can be taken as homogeneous within a certain type, say, female indigenous children living in rural areas.

Therefore, in view of the fact that we adopt the CA in defining wellbeing as the freedom to achieve, which is in turn represented by the capability set, we would like to posit the idea that capability sets (i.e. potential functionings) and not achieved functionings or outcomes should be the object of equalization in the quest for social justice. We stand by this argument, even if capability sets are heterogeneous and may not be directly observed due to their 'potential' nature, thus posing a formidable challenge for practical purposes. Policy assessment and design based only on EOp leaves important theoretical gaps concerning suitable answers to our first question.

\section{Which social states are better/more just and should be promoted by public action?}

Once one establishes that the appropriate concept of wellbeing is the capability set, it is clear that an expansion and, ideally, an equalization of individuals' capability sets should be the ultimate social objective for development. Turning to the second question that guides us through this paper, the CA, by itself, falls short; it does not go further to elaborate on actual ways of achieving these goals. Sen (2009) himself has stated:

"The capability perspective does point to the central relevance of the inequality of capabilities in the assessment of social disparities, but it does not, on its own, propose any specific formula for public decisions" (p. 232). 
The CA's major strengths, namely the broadness and the context-based character of the framework that it offers, might just be its weakness in terms of its lack of possible suggestions on what can or should be done to increase social justice. The CA limits itself to advocating for the equalization of capabilities as a normative rule for social justice, implying that a fair distribution of potential functionings amongst members of a society ought to be the public goal (Fleurbaey, 2007). The need for further elements other than those depicted in the CA is evident for giving a plausible answer to our second question.

We stress that these elements cannot come from traditional normative prescriptions inspired from neoclassical welfare economics. Although they are deeply influential for choosing the 'right' social states, these prescriptions rely heavily on the extrapolation of the notion of individual utility to that of social utility. General equilibrium modeling fits well as a widely known example of such practice (Devarajan \& Robinson, 2002). This Arrovian view on social choice has also been criticized for having a thin informational basis, focusing exclusively on individuals' satisfaction or happiness for the maximization of some aggregation of the latter, prior to the search for the best allocations of lifestyle outcomes. Because of these facts, we argue that traditional normative and theoretical schemes from neoclassical welfare economics are clearly unsuitable supplements to the CA in the sense described in the introductory paragraphs by Robeyns (see the quotation in Section 1, with own emphasis).

According to Pignataro (2012), the last 15 years have witnessed a notable revolution in terms of thinking about social justice and what should be perceived as a fair social state and how to promote it. Roemer's EOp (Roemer, 1998) stands out in this evolution of thinking and in our opinion provides a suitable supplement to the CA. In the remainder of this section, let us present ordered arguments supporting this idea and formally identify the elements of the EOp that are compatible with the CA's rationale.

The principal ideal of EOp is to achieve a social state in which circumstances do not bear any influence on people's outcomes, either directly or indirectly through influences over the efforts people make to achieve them. This irrelevance of circumstances is a condition for a Romerian leveling of the playing field conception of social justice. This just society may be utopic, but this way of thinking has undeniably shed a clear light on plausible governmental interventions to promote this ideal. For instance, Roemer et al. (2003) have assessed the extent to which reigning fiscal regimes in eleven countries contribute to equalizing opportunities for income acquisition based on the EOp approach. Also based on this approach, but within a different empirical framework and operational model, Betts and Roemer (2005) have analyzed the contribution of an educational finance reform in the USA to the equalization of opportunities for education. Indeed, in the EOp there exists a notion of optimal public policy for social justice, and it is one entailing equality of opportunities to achieve a certain outcome.

Today, the discussion on the selection of such socially optimal public policies is of great importance in academic circles (Pignataro, 2012). According to Ramos and Van de Gaer (2012), there are two conflicting practical programs for identifying EOp optimal public policies. The first was proposed by Roemer himself in 1998 and aims to eliminate inequality of outcomes for individuals exerting the same effort, regardless of their type. This program is called ex-post equality of opportunity.

Mathematically, the operationalization of ex-post equality of opportunity builds directly on Rawls' prescription of focusing on the least advantaged people. According to the ex-post EOp, the optimal public policy is formed by a set of specific public actions $p_{\pi}$, where $\pi$ is a centile of the effort distributions of all types, so that every $p_{\pi}$ maximizes the outcome of the least 
advantaged individuals having exerted the same degree of effort $\pi$ in all types. Using the notation introduced before, this is formalized by:

$$
\max _{p_{\pi}} \min _{\text {across types }} y_{t}\left(\pi, p_{\pi}\right)
$$

Evidently, as it is impractical to find an optimal public policy for every centile, Roemer invokes outcome-based tools to define a single optimal public policy compatible with social justice, $p$, as the one that helps achieving maximization of the average advantage of the least favored across every degree of effort. Formally, this policy solves the following optimization program:

$$
\max _{p} \sum_{\text {every } \pi} \min _{\text {across types }} y_{t}\left(\pi, p_{\pi}\right) \quad \text { or } \max _{p} E\left[\min _{t} y_{t}\left(\pi, p_{\pi}\right)\right]
$$

The arguments that we have presented make it clear that this program is too heavily centered on outcomes, leading to clear theoretical incompatibilities with the CA. As the ex-post program is the original program proposed by Roemer, this is perhaps one of the reasons economic and human development scholars have neither insistently pushed forward to build a mix of Sen's and Roemer's theories, nor searched for empirical endeavors founded on such a possible mix. In effect, this program compares outcomes across individuals and explicitly seeks to pull the least advantaged individuals upwards, identifying these people based on their outcomes. The ex-post framework follows the compensation principle, suggesting public policies that contribute to the equalization of outcomes for individuals exerting the same level of effort under different circumstances (Pignataro, 2012).

There is, however, a second program for achieving equality of opportunity, whose advancement is generally attributed in the literature to Van de Gaer (see e.g. Van de Gaer (1993); Ramos \& Van de Gaer (2012)) and which is concerned with the equalization of prospects of outcomes for every individual, regardless of their type. This program is called ex-ante equality of opportunity and it advocates in favor of a policy allowing the maximization of the prospects of outcomes (potential outcome) of the least advantaged type in a society, as measured by the average of the outcomes over all the members of that type:

$$
\max _{p} \min _{\text {across types }} \sum_{\pi} y_{t}\left(\pi, p_{\pi}\right) \quad \text { or } \max _{p} \min _{\text {across types }} E_{\pi}\left[y_{t}\left(\pi, p_{\pi}\right)\right]
$$

Even if the formalization of this program differs only on the inversion of the sum and min operators, the implications for a conceptual compatibility between the EOp approach and the CA are of paramount importance. In the ex-ante approach, the degree or level of effort are to be left unobserved, as was proposed in the original Romerian EOp conception, but remains what ultimately determines the differences of outcomes within a type; it respects individual heterogeneity. The ex-ante program builds on the reward principle of analyzing differences in outcomes for individuals under the same circumstances (Pignataro, 2012). Implicitly, there should not be any kind of public intervention attempting to equalize outcomes that originate from each individual's responsibility.

Therefore the answer to our second question is that public policies should foster equality of opportunity, conceived as a situation where sets of possible outcomes are the same for every type within a society. Furthermore, policy priority is given to pulling up the least-advantaged type distribution of outcomes and for that, it is a Rawlsian conception of equality of opportunity.

\section{Combination}

Based on the preceding discussion, we propose to deepen the consideration of individual capability sets as the multidimensional unobserved outcome of an EOp-based theoretical framework in the quest for policymaking for wellbeing with justice. We believe that this constitutes another step forward in the operationalization of the CA for policymaking. 
We propose the conceptual scheme in Figure 3 below to represent the theoretical linkages that will be highlighted in this section.

Figure 3: Scheme of a Capability Approach-Based Equality of Opportunity Framework

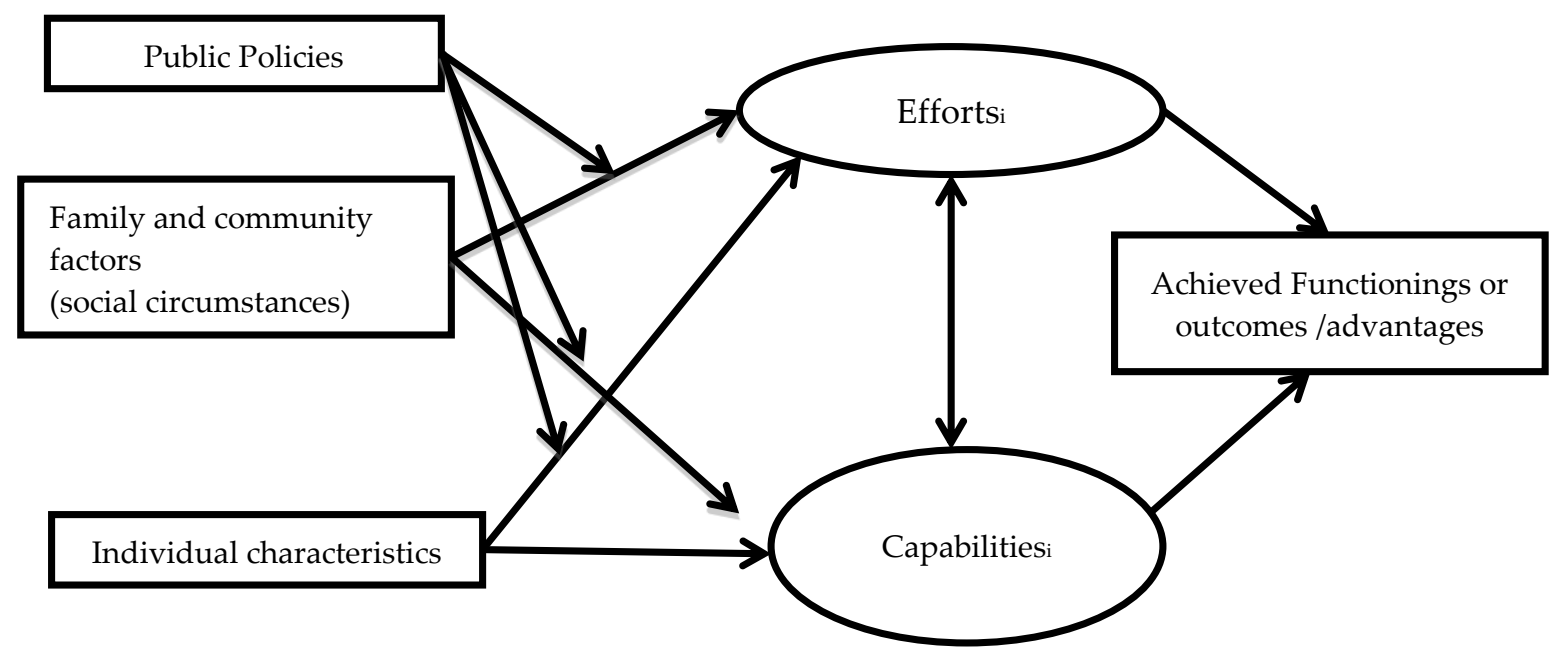

Let us start our reasoning and the explanation of the scheme recalling that wellbeing, measured by the capability set, is multidimensional and intrinsically unobservable due to its counterfactual nature and, according to EOp, it is determined by individual efforts, which are also unobservable and reflect an individual sense of responsibility. Thus efforts are essential for understanding wellbeing, as is emphasized by Roemer throughout his work (Roemer, 1998; Roemer et al., 2003; see also Betts \& Roemer, 2005). Nevertheless, in most empirical EOp-based work the amount of effort exerted by a person has been treated as a residual error term, with no further consideration for its estimation (see for example Bourguignon et al., 2007; Cogneau \& Gignoux, 2008; Roemer et al., 2003). Therefore the combination that we propose introduces a causal relationship between unobservable or latent variables that remains empirically understudied in public policy and economic development literature and we make the case that it can deliver important results for understanding and assessing the role of public policies for wellbeing.

We postulate the existence of a two-way relationship between capabilities and efforts, which together lead to multiple achieved outcomes or functionings, thus capturing the CA's multidimensional essence. We argue that efforts are shaped by capabilities by virtue of the individual nature of the decision-making process on efforts. We reason that potential feasible lifestyle outcomes of a person configure the practical choices and actions she makes in order to achieve the outcomes that suit her best, according to her needs, tastes and desires. Efforts, in turn, can affect capabilities by bringing in more potential outcomes within the capability set, expanding lifestyle possibilities for people to choose from.

Both efforts and capabilities are in turn influenced by individual characteristics and surrounding features, called circumstances. These include: i) individual characteristics such as gender, ethnicity and age; ii) other multilevel aspects concerning a) family and household, such as the composition, living conditions, religion and culture and b) community, such as the environment, the language spoken, or the level of local economic development. In our combination, public policies are also a part of the environment as the CA mandates, and according to the ex-ante framework of the EOp such policies should aim at equalizing opportunities by reducing the role played by circumstances beyond one's control in the determination of capabilities and efforts. Thus public policies may not explicitly shape 
circumstances such as gender, household composition or language spoken in the community, but they do shape the extent to which these circumstances affect an individual's capabilities and efforts.

Thus, building on Rawlsian maximin rationale, which states that a society would achieve justice and equality when prospects of life for the least fortunate are as great as they can be (Rawls, 1971, 1999), an optimal policy for equalization of capability sets across types $t=1 \ldots T$ would be given by:

$$
\max _{p} \min _{\text {across types }} \sum_{i \in \text { type } t} Q_{i, t} \text { or } \max _{p} E Q_{\min }
$$

where $Q_{i, t}$ is the capability set of the $i$-th individual belonging to type $t$ and $E Q_{\min }$ is the average capability set of the least advantaged type.

Here we would like to recall some key equations introduced by Sen (1985) for defining capabilities so that we are clear as to what is being maximized. Let $x_{i}$ be the commodity vector possessed by an individual $i$. The individual makes use of the characteristics of the commodities in order to 'convert' the resources into a functioning, i.e a 'being' or a 'doing'. We take the term 'commodity' in a large sense, including personal resources as well as social and institutional infra-structural support or circumstances, i.e all that enters the conversion process. The conversion function is written as:

$$
\mathrm{b}_{\mathrm{i}}=f_{i}\left(c\left(x_{i}\right)\right)
$$

Note that given the same $x_{i}$ achieved functioning can be different from one individual to another due to the dependence of $f$ on $i$ as personal characteristics such as age, gender, health status, tastes, and the effort put in by the individual, all of which play an important role in the conversion process. The set of all functionings that can be potentially achieved using the commodity vector is called the capability set:

$$
Q_{i}=\left\{b_{i} \mid b_{i}=f_{i}\left(c\left(x_{i}\right)\right) \text {, for some } f_{i} \text { and for some } x_{i}\right\}
$$

Thus we are not maximizing utility in (7), neither are we maximizing resources or outcomes (achieved functionings). It is indeed the set of feasible functionings of an individual, in other words her freedom to achieve valued things in life, which we seek to maximize in our approach.

In our setting, each individual $i$ belongs to a type $t$ and hence every individual will have a double index $i, t$ from now on. As expressed in equation (7), our optimal policy for wellbeing with justice is one which maximizes the capability sets (feasible outcomes) for the least favored. In our model, the capability set depends on personal circumstances and efforts, and the efforts themselves can in turn be influenced by circumstances. Thus

$$
Q_{i, t}=Q_{i, t}\left(x_{i, t}, e_{i, t}\left(x_{i, t}\right)\right)
$$

where circumstances are included in $x_{i, t}$ and $e_{i, t}$ denotes the effort. In Roemer's reasoning, the most disadvantaged type identifies the least favored and hence it represents a group rather than a single individual. Thus we take the expected feasible outcome (or the expected capability set) of the least favored type as our objective function in order to be consistent with the ex-ante EOp approach. Then the optimization program can be written as:

$$
\max _{p} \min _{\text {across types }} E\left[Q_{i, t}\left(x_{i, t}, e_{i, t}\left(x_{i, t}\right)\right) \mid x_{i, t}\right]
$$

For simplicity of notation, let us denote the expectation as:

$$
E Q_{i, t} \equiv E\left[Q_{i, t}\left(x_{i, t}, e_{i, t}\left(x_{i, t}\right)\right) \mid x_{i, t}\right]
$$

and the minimum as: 


$$
E Q_{i, \min } \equiv \min _{\text {across types }} E Q_{i, t}
$$

In a very general setting, $E\left[Q_{i, t}\left(x_{i, t}, e_{i, t}\left(x_{i, t}\right)\right) \mid x_{i, t}\right]$ can be different from one individual to another and hence we keep the index $i$ in the expectation. Thus the 'optimal' policy will be given by $p^{*}{ }_{i, \min }$ such that $E Q_{i, \min }$ is maximum ${ }^{2}$, i.e. we should have $\frac{\partial E Q_{i, \min }}{\partial p}=0$ at $p=p^{*}{ }_{i, \min }$. Note that the solution could also vary from one type to another. Thus, $p^{*}{ }_{i, \min }$ denotes the optimal policy applied to an individual $i$ belonging to the least favored type ${ }^{3}$.

Now, rather than deriving the optimal policy, if we want to see how effective a policy is in promoting EOp (or reducing inequality of opportunity) following Roemer's idea, we can evaluate to what extent the policy is able to change the impact of a generic circumstance variable $x_{i, \min }$ of an individual $i$ belonging to the least favored type on her expected capability set $E Q_{i, \min }$. The effect of circumstance for the least favored type can be computed as:

$$
\frac{\partial E Q_{i, \min }}{\partial x_{i, \min }}=\frac{\partial E Q_{i, \min }}{\partial x_{i, \min }}+\frac{\partial E Q_{i, \min }}{\partial e_{i, \min }} \frac{\partial e_{i, \min }}{\partial x_{i, \min }}
$$

where the derivatives are to be calculated using equation (10). Let us denote:

$$
\begin{aligned}
& \frac{\partial E Q_{i, \text { min }}}{\partial x_{i, \text { min }}}=\frac{\partial E Q_{i, \text { min }}}{\partial x_{i, \text { min }}}\left(p_{i, \text { min }}\right) \equiv g_{i, \text { min }}\left(p_{i, \text { min }}\right) \\
& \frac{\partial e_{i, \text { min }}}{\partial x_{i, \text { min }}}=\frac{\partial e_{i, \text { min }}}{\partial x_{i, \text { min }}}\left(p_{i, \text { min }}\right) \equiv h_{i, \text { min }}\left(p_{i, \text { min }}\right)
\end{aligned}
$$

The first function above depicts the direct impact of circumstances on expected capability sets and the second depicts their indirect impact through effort. Thus the total effect of circumstances as a function of public policies is given by:

$$
\frac{\partial E Q_{i, \min }}{\partial x_{i, \min }}=g_{i, \min }\left(p_{i, \min }\right)+\frac{\partial E Q_{\min }}{\partial e_{i, \min }} h_{i, \min }\left(p_{i, \min }\right) \equiv k_{i, \min }\left(p_{i, \min }\right)
$$

Therefore, the condition that a public policy must fulfill in order to be EOp-optimal, i.e. to nullify the effect of circumstances on expected capability sets for the least favored type, is given by:

$$
k_{i, \min }\left(p^{*}{ }_{i, \min }\right)=0
$$

denoting this optimal policy as $p^{*}{ }_{i, m i n}$.

Now, if we would like to derive the optimal policy for any type $t$ in general and not necessarily the least favored one, we would just need to replace 'min' by ' $t$ ' in the above derivation and the optimal policy would then be given by $p^{*}{ }_{i, t}$ such that:

$$
k_{i, t}\left(p^{*}{ }_{i, t}\right)=0
$$

with

$$
k_{i, t}\left(p_{i, t}\right) \equiv \frac{\partial E Q_{i, t}}{\partial x_{i, t}}=g_{i, t}\left(p_{i, t}\right)+\frac{\partial E Q_{i, t}}{\partial e_{i, t}} h_{i, t}\left(p_{i, t}\right)
$$

As an illustration we present a case in which the above relations take a linear form, the policy value is common to all individuals belonging to a given type, and the coefficients are also invariant within a given type. Let

$$
E Q_{i, t}=x_{i, t}^{\prime}\left(A_{t}+B_{t} p_{t}\right)+e^{\prime}{ }_{i, t}\left(C_{t}+D_{t} p_{t}\right)+z^{\prime}{ }_{i} F_{t}
$$

\footnotetext{
${ }^{2}$ A policy $p$ has an impact on wellbeing through its influence on the effect of circumstance variables on capabilities or efforts (see later).

${ }^{3}$ In practice, the policy variable will probably take the same value for all individuals belonging to the same type. However, here we present a very general theoretical setting in which it can vary from one individual to another.
} 


$$
e_{i, t}=x^{\prime}{ }_{i, t} G_{t}+z_{i}^{\prime} H_{t}
$$

(where $z^{\prime}{ }_{i}$ represents a vector of personal characteristics)

Then,

$$
\frac{\partial E Q_{i, t}}{\partial x_{i, t}}=g_{i, t}\left(p_{t}\right)+\frac{\partial E Q_{i, t}}{\partial e_{i, t}} h_{i, t}\left(p_{t}\right) \equiv k_{i, t}\left(p_{t}\right)=A_{t}+B_{t} p_{t}+G_{t}\left(C_{t}+D_{t} p_{t}\right)
$$

and the optimal policy is $p_{t}^{*}$ such that:

$$
A_{t}+B_{t} p_{t}^{*}+G\left(C_{t}+D_{t} p_{t}^{*}\right)=0
$$

Let us stress that the observable aspects of a person's lifestyle, call them either outcomes or functionings, are not the essence of the optimization program. The reason is two-fold. First, they are not a person's wellbeing according to the CA but only partial manifestations of her wellbeing. Second, a person's actual lifestyle is ultimately a personal choice. In other words, we advocate for a theoretical framework in which public policies do not directly aim at influencing a person's lifestyle but act in an indirect way by promoting the enabling factors of capabilities and efforts (potential lifestyles), leaving the actual choice to the individual, thus respecting her freedom.

In order to clarify the difference between the proposed theoretical framework and separate EOp and CA-based theoretical frameworks, let us consider the following example. There are important public policies, more precisely social policies, built around conditional cash transfers for students, that aim to diminish rates of school abandonment, increase their academic performance and ultimately, their wellbeing and promote justice within society (Bolivia's Bono Juancito Pinto is an example of such a policy, see Yañez, 2012). In this concrete case, the two questions that have guided our discussion could take the following form:

1) What are we making less unequal through this conditional transfer?

2) What is the adequate amount for the cash transfer to promote equality?

A plausible answer to the first question from a 'pure' EOp perspective would state that one needs to identify one observable outcome or advantage enjoyed by the beneficiaries that has a link to the cash transfer, say the schooling gap, defined as the difference between the years of schooling a person should have given her age and her actual years of schooling. This variable would constitute the proxy of opportunities for education and ultimately wellbeing, which is what the cash transfer aims at equalizing.

A traditional EOp-based theoretical framework adopting an ex-ante program for equality of opportunity would bring about an answer to the second question stressing that the cash transfer has a direct impact on the expected schooling gap. It would go on to advocate for seeking an amount of the transfer so that expected schooling gap is minimized (i.e. opportunities for education are maximized) for the least favored type of people, say indigenous females. Within this type, schooling gap differences, i.e. wellbeing differences, would then be the absolute responsibility of the beneficiaries or fruit of their efforts, as they all are supposed to share the same circumstances.

A traditional CA-based framework would stress that schooling gap is a rather oversimplified proxy of the beneficiaries' wellbeing and that the latter should be measured, for instance, as their capability to be educated (not to be confused with individuals' inherent abilities like intelligence). A single observed outcome for gauging such a broad concept is clearly insufficient and unnecessary; the policy might also influence other aspects of wellbeing that can be observed, such as the ability to read and to understand abstract logic reasoning (by increasing the possibility of buying more books using the extra cash and/or hiring a tutor) or the ability to read foreign languages (by increasing the possibility of entering a language institute). The CA would go on to stress that the actual schooling gap is, to some extent, a choice among other feasible 
options of the beneficiaries. Thus it would suggest only an indirect impact of the cash transfer on the schooling gap, through the expansion of the beneficiaries' capability to be educated.

However, due to the absence of any prescriptions regarding how to go about achieving equality of opportunity in these multidimensional aspects, the CA does not give a plausible answer for the second question apart from the statement: the greater the capability to be educated for all beneficiaries, the better.

The combination that we propose would in fact lead to a precise and operational answer to both the questions combining remarkable elements of both approaches. To answer the first question, the combined approach would advocate for the multiplicity of outcomes for gauging wellbeing, inheriting the logic of the CA that we just presented. Not only would our approach take into account the multidimensional aspect of wellbeing, but it would also respect the beneficiaries' freedom to choose, suggesting only an indirect influence of the transfer over all outcomes.

To answer the second question, our combination would inherit the rationale of the EOp, arguing in favor of the equalization of capability to be educated by searching for an amount of the transfer that maximizes this capability for the 'least capable' across different types and ideally annuls the effect of circumstances on the capability of being educated. The combination would not suggest an equalization of any outcome, in respect of individual's freedom to choose. Furthermore, the search of this adequate amount of transfer would not let effort be considered as a residual variable; it is an important determinant of actual outcomes. Effort would be, at least, partially gauged by some observable indicators, such as daily hours of study and frequency of visits to the school library. Thus effort would not be considered as an absolute personal responsibility; each of the effort indicators that we mention is surely influenced by the beneficiaries' capability to be educated and the converse is also true.

\section{Concluding Remarks}

The quest for improved collective wellbeing with justice has always been a priority for most of the economic and political constructs that we know of. In this paper, we have attempted to show that there exist powerful philosophical and theoretical frameworks that contain important elements, which can and should be combined to come up with operational answers to this question. In particular, we rely on two theories that have recently gained an overwhelmingly increasing visibility in academic and policy circles, namely the Capability Approach and the Equality of Opportunity approach.

One of the main contributions we want to make in this paper is to put on the table some ordered arguments for the fact that neither of these leading theories alone possesses sufficient theoretical elements to guide policymaking for wellbeing with justice. Thus we advocate for a theoretical construction that builds on these two approaches to provide insight on how to design and evaluate policies for improvement of wellbeing with social justice. We show how the similarities and differences between the two approaches are complementary to each other and how they can help to set a solid theoretical basis for the assessment and design of public policies that seek to improve social justice. Consequently, there are ways to explore the issue without having to build an entirely new approach. The particular manner in which we propose to combine the two theories has, to our knowledge, not been explored in the related literature.

We show that the ex-post framework of the Equality of Opportunity approach, which is its original conception, is too heavily centered on people's lifestyle outcomes, with too little theoretical consideration for individual heterogeneity and choice. However, the ex-ante framework of the Equality of Opportunity approach depicts elements that are compatible with 
the logic of the Capability Approach, in the sense that both respect individual heterogeneity and freedom, and do not aim at equalization of results as a social justice paradigm.

In the combination that is proposed, we argue for the need to explicitly consider the role of efforts while analyzing public policies and their link to wellbeing and justice. Indeed, the line of reasoning that we present makes the case that individual efforts influence and are influenced by individual capabilities, but to our knowledge, there are no theoretical attempts in the existing literature to relate these two key variables in the understanding of wellbeing. Efforts have often been treated as a residual variable in most empirical applications of the Equality of Opportunity approach, due to its unobservable nature. We stress that this is not an insurmountable obstacle as, even if potential achievements cannot be observed due to their counterfactual nature, there are empirical endeavors that have successfully taken account of this feature in their operationalization methodology. A similar approach can also be followed for operationalizing 'effort'. In other words, technical difficulties for gauging bidirectional causal relations between unobservable variables should not hinder theoretical developments that can shed important light for understanding wellbeing and its relation to public policies.

Therefore, while designing and assessing public policies, one needs to target and equalize capability sets of individuals, rather than their outcomes. This is done through a maximin algorithm that maximizes the capability set of the group that is the least favored in terms of circumstances while taking into account the influence of circumstances and policies on capabilities and efforts, and explicitly incorporating interdependencies between the latter two variables. We define this 'well-being with justice' problem in a technical way and discuss the solution from an optimal public policy angle.

We are aware that the main challenges for the empirical application of our theoretical framework concern the development of a rigorous econometric model that helps to identify the complex structural relations that we have proposed in this paper, as well as the existence and quality of adequate information for its implementation. Nevertheless, we believe that these challenges should be faced and we intend to attempt a practical implementation of our framework in a future study, as we are convinced that the theoretical discussion, although enriching, needs to be strengthened by empirical investigations.

\section{Authors}

Jaya Krishnakumar

University of Geneva

Ricardo Nogales

University of Geneva

Publishing Timeline

Received 31 October 2014

Accepted 26 February 2015

Published 30 July 2015

\section{References}

Alkire, S. (2013). Well-Being, happiness and public policy. OPHI Research in Progress Series 2013, University of Oxford. http://www.ophi.org.uk/wp-content/uploads/OPHI-RP-37a2013.pdf?0a8fd7

Alkire, S. \& Foster, J. (2011). Counting and multidimensional poverty measurement. Journal of Public Economics, 95(7-8), 476-487. http://dx.doi.org/10.1016/j.jpubeco.2010.11.006 
Anand, P., Hunter, G., Carter, I., Dowding, K., Guala, F. \& Van Hees, M. (2009). The development of capability indicators. Journal of Human Development, 10(1), 125-152. http://dx.doi.org/10.1080/14649880802675366

Anand, P., Hunter, G. \& Smith, R. (2005). Capabilities and wellbeing: Evidence based on the Sen Nussbaum approach to welfare. Social Indicators Research, 74(1), 9 - 55. http://dx.doi.org/10.1007/s11205-005-6518-z

Anand, P., Krishnakumar, J. \& Tran B. (2010). Measuring welfare: Latent variable models for happiness and capabilities in the presence of unobservable heterogeneity. Journal of Public Economics, 95(3-4), 205-215. http://dx.doi.org/10.1016/j.jpubeco.2010.11.007

Anand, P., Santos, C. \& Smith, R. (2007). The measurement of capabilities. In Festschrift for Amartya Sen, (Basu \& Kanbur, eds), Oxford, Oxford University Press.

Anand, P. \& van Hees, M. (2006). Capabilities and achievements: An empirical study. The Journal of SocioEconomics, 35, 268-284. http://dx.doi.org/10.1016/j.socec.2005.11.003

Anand, S. \& Sen, A. (1993). Human Development Index: Methodology and measurement. Human Development Report Office, Occasional Paper 12, UNDP, New York.

Arneson, R. (1989). Equality and equal opportunity for welfare. Philosophical Studies, 56, 77-93. http://dx.doi.org/10.1007/BF00646210

Betts, J. \& Roemer, J. (2005). Equalizing opportunity for racial and socioeconomic groups in the United States through educational finance reform. Economics Working Paper Series, University of California at San Diego, Department of Economics.

Blackorby, C. \& Bossert, W. (2008). Interpersonal comparisons of well-being. in The Oxford Handbook of Political Economy, (Wittman \& Weingast, eds.), Oxford Handbooks Online, Oxford. http://dx.doi.org/10.1093/oxfordhb/9780199548477.003.0023

Bourguignon, F., Ferreira, F. \& Menéndez M. (2007). Inequality of opportunity in Brazil. Review of Income and Wealth, 53(4), 585-618. http://dx.doi.org/10.1111/j.1475-4991.2007.00247.x

Brunori, P., Ferreira, F. \& Peragine, V. (2013). Inequality of opportunity, income inequality and economic mobility: Some international comparisons. Policy Research Working Paper 6304. The World Bank. http://dx.doi.org/10.1596/1813-9450-6304

Chiappero Martinetti, E. (2009). Poverty as lack of opportunity: A comparison between John Roemer and Amartya Sen. Rivista Italiana di Economia, Demogra_a e Statistica, 73(1-2), 71- 90.

Chiappero Martinetti, E. \& Salardi, P. (2008). Wellbeing process and conversion factors: An estimation. HDCPIRC Working Paper Series, 3, ISSN 1974-1952. Human Development and Poverty International Research Center. http://www.ophi.org.uk/wp-content/uploads/ssChiappero-Martinetti-Salardi2008.pdf

Cogneau, D. \& Gignoux, J. (2008). Earnings inequality and education mobility in Brazil over two decades. In Poverty, inequality and policy in Latin America, (Klasen \& Nowak-Lehmann, eds.). MIT Press, USA.

Comim, F. (2001). Operationalizing Sen's Capability Approach. Paper Prepared for the Conference on Justice and Poverty: examining Sen's Capability Approach, Cambridge, 5-7 June 2001. http://www.researchgate.net/publication/239922491 Operationalizing Sen\%27s Capability Approac $\underline{\mathrm{h}}$

Devarajan, S. \& Robinson, S. (2002). The influence of computable general equilibrium modeling on policy. Trade and Macroeconomics Division-TMD Discussion Paper 98, International Food Policy Research Institute, Washington, USA.

Di Tommaso, M. L. (2007). Children's capabilities: A structural equation model for India. The Journal of Socio-Economics, 36, 436-450. http://dx.doi.org/10.1016/j.socec.2006.12.006

Ferreira, F. \& Gignoux, J. (2008). The measurement of inequality of opportunity: Theory and application to Latin America. Journal of Income and Wealth, 57(4), 622-257. http://dx.doi.org/10.1111/j.14754991.2011.00467.x

Fleurbaey, M. (2007). Social choice and the indexing dilemma. Social Choice and Welfare, 29(4), 633-648. http://dx.doi.org/10.1007/s00355-007-0248-x

Haq, M. (1999) Reflections on human development. Oxford University Press, Delhi. 
Igersheim, H. (2006). A.K. Sen et J.E. Roemer : une même approche de la responsabilité?. Working Papers of BETA 2006-08, Bureau d'Economie Théorique et Appliquée, UDS, Strasbourg. http://www.researchgate.net/publication/23693500 A.K. Sen et J.E. Roemer une mme approche de la responsabilit

Jacquet, L. \& Van de Gaer, D. (2011). A comparison of optimal tax policies when compensation or responsibility matter. Journal of Public Economics, 95(11), 1248-1262. http://dx.doi.org/10.1016/j.jpubeco.2010.05.005

Kabeer, N. (2010), Can the MDG's provide a pathway for social justice? The challenge of interesting inequalities. UNDP, New York.

Krishnakumar, J. (2007). Going beyond functionings to capabilities: An econometric model to explain and estimate capabilities. Journal of Human Development, 8(1), 29-63. http://dx.doi.org/10.1080/14649880601101408

Krishnakumar, J. \& Ballón, P. (2008). Estimating basic capabilities: A structural equation model applied to Bolivia. World Development, 36(6), 992-1010. http://dx.doi.org/10.1016/j.worlddev.2007.10.006

Krishnakumar, J. \& Wendelspeiss, F. (2011), The impact of oportunidades on inequality of opportunity in rural and urban areas in Mexico. Working Paper, University of Geneva, Switzerland.

Kuklys, W. (2005). Amartya Sen's Capability Approach: Theoretical insights and empirical applications, Springer, Berlin.

Ministerio de Planificación del Desarrollo (2006). Plan Nacional de Desarrollo: Bolivia digna, soberana, productiva y democrática para Vivir Bien. República de Bolivia. Ed. Plural, Bolivia.

Pignataro, G. (2012). Equality of opportunity: Policy and measurement paradigms. Journal of Economic Surveys, 26(5), 800-834. http://dx.doi.org/10.1111/j.1467-6419.2011.00679.x

Ramos, X. \& Van de Gaer, D. (2012). Empirical approaches to inequality of opportunity: Principles, measures, and evidence. IZA Discussion Papers 6672, Institute for the Study of Labor (IZA).

Ravallion, M. (2010). Mashup indices of development. Policy Research Working Papers, World Bank. http://dx.doi.org/10.1596/1813-9450-5432

Ravallion, M. (2012). On multidimensional indices of poverty. Journal of Economic Inequality, 9(2), 235-248. http://dx.doi.org/10.1007/s10888-011-9173-4

Rawls, J. (1971). A theory of justice. Harvard University Press, Cambridge, MA.

Rawls, J. (1999). A theory of justice, revised edition. Harvard University Press, Cambridge, MA.

Robeyns, I. (2003). The Capability Approach: An interdisciplinary introduction. Document prepared for the Third International Conference on The Capability Approach, held in Italy on September 6, 2003.

Robeyns, I. (2006). The Capability Approach in practice. Journal of Political Philosophy, 14(3), 351-376. http://dx.doi.org/10.1111/j.1467-9760.2006.00263.x

Roemer, J. (1998). Equality of opportunity. Harvard University Press, United States.

Roemer, J., Rolf, A., Ugo, C., Johan, F. L, Stephen, P. J., Arnaud, L., ... Zubiri, I. (2003). To what extent do fiscal regimes equalize opportunities for income acquisition among citizens? Journal of Public Policy, 87(3), 539-565. http://dx.doi.org/10.1016/s0047-2727(01)00145-1

Ruggeri-Laderchi, C., Saith, R., \& Stewart, F. (2003). Does it matter that we do not agree on the definition of poverty? A comparison of four approaches. Oxford Development Studies, 31(3), 243-274. http://dx.doi.org/10.1080/1360081032000111698

Schokkaert, E. (2007). The Capabilities Approach. Discussion Paper Series, 07-34, Center for Economic Studies, Katholeike Universiteit Leuven.

Sen, A. K. (1980). Equality of what? In The Tanner Lecture on Human Values, I, 197-220. Cambridge: Cambridge University Press.

Sen, A. K. (1985). Commodities and Capabilities. Amsterdam: North Holland.

Sen, A. K. (1999). Development as freedom. Oxford: Oxford University Press.

Sen, A. K. (2009), The idea of justice. Harvard University Press, Cambridge MA.

SENPLADES: Secretaría Nacional de Planificación del Desarrollo (2009). Plan Nacional para el Buen Vivir. República del Ecuador. 
Simon, J., Anand, P., Gray, A., Rugkåsa, J., Yeeles, K. \& Burns, T. (2013). Operationalising the Capability Approach for outcome measurement in mental health research. Social Science \& Medicine, 98, 187-196. http://dx.doi.org/10.1016/j.socscimed.2013.09.019

Ura, K., Alkire, S., Zangmo, T. \& Wangdi, K. (2012). An extensive analysis of GNH Index. The Centre for Buthan Studies. ISBN 978-99936-14-67-8

Vallentyne, P. (2005). Debate: Capabilities vs. opportunities for well-being. Journal of Political Philosophy, 13(3), 359 - 371. http://dx.doi.org/10.1111/j.1467-9760.2005.00227.x

Van de Gaer, D. (1993). Equality of opportunity and investment in human capital. Ph.D. dissertation. KULeuven, Leuven.

Van de Gaer, D., Vandenbossche, J. \& Figueroa, J. L. (2013). Children's health opportunities and project evaluation: Mexico's Oportunidades program. Policy Research Working Paper Series 6345, World Bank. http://dx.doi.org/10.1596/1813-9450-6345

Wendelspeiss, F. (2013). Measuring inequality of opportunities with latent variables. Journal of Human Development and Capabilities, forthcoming. http://www.tandfonline.com/doi/abs/10.1080/19452829.2014.907247

Yalonetzky, G. (2012). A dissimilarity index of multidimensional inequality of opportunity. Journal of Economic Inequality, 10(3), 343-373. http://dx.doi.org/10.1007/s10888-010-9162-z

Yañez, E. (2012). El impacto del bono Juancito Pinto: un análisis a partir de microsimulaciones. Revista Latinoamericana de Desarrollo Económico, 17, 75-112. 